\title{
Proteostasis impairment in ALS
}

\author{
Céline Ruegsegger ${ }^{1,4}$ and Smita Saxena ${ }^{1}$ \\ ${ }^{1}$ Institute of Cell Biology, University of Bern, Baltzerstrasse 4, CH-3012 Bern, Switzerland \\ ${ }^{4}$ Graduate School for Cellular and Biomedical Sciences, University of Bern, $\mathrm{CH}-3012$ \\ Bern, Switzerland
}

Corresponding author: Smita Saxena, Institute of Cell Biology, Baltzerstrasse 4, $\mathrm{CH}-$ 3012 Bern, Switzerland

tel: +4131-6314620, Fax: +4131-6314431, email: smita.saxena@izb.unibe.ch

Pages: $\mathbf{3 1}$

Figure: 1

\section{ACKNOWLEDGEMENTS}

C.R. and S.S. were supported by Swiss National Science Foundation grants: 128460 and 150756 and Frick foundation for ALS research. 


\section{Highlights}

- Basic mechanisms of the ER homeostasis, ubiquitin proteasome system (UPS), and autophagy are overviewed.

- Discussion on how proteostasis impairment occurs in ALS

- Relevance between ER stress and ALS-associated pathomechanisms

- Discuss the roles and dysfunction of autophagy and UPS system in ALS

- Discuss studies wherein amelioration of ALS pathophysiology was achieved by targeting proteostasis dysfunction in ALS 


\section{$\underline{\text { Abstract }}$}

In physiological conditions the maintenance of the cellular proteome is a prerequisite for optimal cell functioning and cell survival. Additionally, cells need to constantly sense and adapt to their changing environment and associated stressors. Cells achieve this via a set of molecular chaperones, protein clearance pathways as well as stress-associated signaling networks which work together to prevent protein misfolding, its aggregation and accumulation in subcellular compartments. These processes together form the proteostasis network which helps in maintaining cellular proteostasis. Imbalance or impairment in this processes is directly linked to ageing associated disorders such as diabetes, cancer, stroke, metabolic disorders, pulmonary fibrosis, inflammation and neurodegenerative diseases. In this review, we provide insights into the proteostasis process and how its failure governs neurodegenerative disorders with a special focus on Amyotrophic lateral sclerosis (ALS). 


\section{Introduction}

In order to be functional, proteins after synthesis need to be folded in a specific and unique three-dimensional tertiary structure. However, about $30 \%$ of the newlysynthetized proteins are misfolded and therefore require refolding (Schubert et al., 2000). Under normal conditions, cells have an efficient protein quality control machinery which is able to detect and handle misfolded proteins. This is accomplished by various chaperones such as heat shock proteins that recognize wrongly folded proteins, help in their refolding, prevent their aggregation and provide aid to repair damaged proteins. In case all attempts to repair and correctly refold the proteins fails then these chaperones also actively mediate their removal. This protein quality control and the maintenance of proteome homeostasis; also termed as proteostasis is a fundamental process required to keep cells healthy and functional. As proteins are only slightly stable at physiological temperatures they are constantly exposed to the high probability of misfolding and therefore the process of cellular proteostasis is highly demanding (Hipp et al., 2014). Proteostasis deficits increase with age and have been shown to facilitate the development and progression of various diseases, including neurodegenerative diseases (NDs), (see Fig. 1), (Ciechanover and Brundin, 2003; Hartl et al., 2011; Hipp et al., 2014; Takalo et al., 2013).

The pathogenesis of NDs centrally involves abnormal accumulation and aggregation of specific misfolded proteins in distinct regions of the brain (Davies et al., 1997; Koo et al., 1999; Martindale et al., 1998; Takalo et al., 2013). For this reason, NDs are viewed as cerebral proteopathies, in which the accumulation of particular misfolded protein aggregates is a key causative factor (Frost and Diamond, 2010; Golde and Miller, 2009; Haass and Selkoe, 2007; Saxena and Caroni, 2011; Stoppini et al., 2004). Further, there is increasing evidence suggesting that genetic mutations or environmental factors can provoke or accelerate protein misfolding and aggregation in NDs (Takalo et al., 2013). Proteins that are known to be involved in NDs like $A \beta$, tau, $\alpha$-synuclein and poly $Q$ expanded proteins, possess few stable three-dimensional structures in physiological conditions and are prone to misfolding. It is well accepted that specific neuronal populations are selectively affected but how those misfolded proteins eventually disrupt 
particular cellular protein networks, thereby leading to selective neuronal vulnerability in NDs remains unresolved (Brignull et al., 2006; Saxena and Caroni, 2011).

In normal conditions a large majority of proteins are made up of structured and ordered domains which are connected together by flexible linkers. In contrast, NDs causing proteins such as a-synuclein, A-beta, Tau, and Polyglutamine expanded proteins are intrinsically disordered and largely unstable in physiological conditions. If misfolded these proteins expose beta sheets which are prone to aberrant interactions with other important protein complexes thereby deregulating multiple signaling pathways (Gidalevitz et al., 2006); (Hartl et al., 2011; Hetz et al., 2015; Hipp et al., 2014; Roth and Balch, 2011). Moreover, these beta sheet-aggregates are largely resistant to proteolytic pathways and eventually lead to the formation of inclusion bodies or extracellular plaques. Importantly, each ND specific protein impairs a unique cellular signaling network thereby contributing to proteostasis impairment and selective neuronal vulnerability in NDs. The identity and understanding of these signaling pathways whose impairment governs the well observed selective neuronal vulnerability is critical for the understanding of mechanisms associated with NDs.

Ageing is considered to be a major risk factor for NDs (Cuanalo-Contreras et al., 2013; Kikis et al., 2010). It has been shown that insoluble proteins accumulate in all tissues during aging in different species (Cuanalo-Contreras et al., 2013; David et al., 2010; Kikis et al., 2010). What is not clear is whether the age-dependent accumulation of those insoluble proteins is the cause for cellular dysfunction leading to aging or a consequence of reduced proteostasis during aging (Cuanalo-Contreras et al., 2013). Using C. elegans as a model system for ageing, several hundreds of proteins with diverse functions were shown to become highly insoluble with age and misfold (David et al., 2010; ReisRodrigues et al., 2012). Notably, small molecules against specific misfolded proteins restored protein homeostasis in vivo and increased life span, consistent with the relationship between protein aggregation and aging (Alavez et al., 2011; Alavez and Lithgow, 2012). 


\section{Protein Quality Control Pathways}

We first briefly describe the relevant pathways involved in proteostasis maintenance and later discuss how those pathways are perturbed in motoneurons in ALS and provide examples implicating proteostasis dysfunction in the etiology of ALS.

\section{ER stress and the UPR Pathway}

The endoplasmic reticulum (ER) is an important subcellular compartment playing a major role in protein biosynthesis and secretion. This membranous organelle is spread throughout the internal volume of the cell, and has specialized contact points with other membrane organelles such as the nucleus, the Golgi, mitochondria, lysosomes, and the plasma membrane. Three different major cellular processes regularly occur in the ER lumen; (1) protein folding and secretion, (2) lipid biogenesis and (3) calcium storage and release (Shore et al., 2011). Proteins that are synthesized in the rough ER undergo glycosylation followed by oxidative folding through the Calnexin / Calreticulin chaperone system which also involves ER resident protein 57 (ERp57). This process is essential for a large majority of proteins as it not only helps in the folding of proteins but also provides higher stability of the polypeptides (Frickel et al., 2004; Hetz and Mollereau, 2014; Oliver et al., 1997; Oliver et al., 1999). Then the glycosylated proteins that are correctly folded are transported from the ER to the Golgi apparatus, where further maturation takes place before they are distributed to their final localization. However, the folding process in the ER is imperfect and a fraction of the proteins never reach their proper native conformation. The incorrect folded polypeptides must dislocate to the cytosol where they are degraded via the ubiquitin-proteasome system, a process that is mediated by ERassociated protein degradation (ERAD) protein complexes (Hetz and Mollereau, 2014). The flux of unfolded polypeptide chains entering the ER to be folded by chaperones is varying and the cells constantly sense and adjust the ER folding capacity depending on demand, thereby assuring the high folding quality of proteins. In case the protein folding demand is too high for the cell then the accumulation of misfolded proteins within its lumen is the most important source of stress to the ER (Ron and Walter, 2007). The conserved signal transduction pathway that has stress sensors facing the ER lumen is 
activated, leading to the Unfolded Protein Response (UPR) (Hetz et al., 2015; Ron and Walter, 2007). These ER associated stress sensors play a key role in coordinating cell adaptions to altered physiological demands and in response to other cellular stressors (Bernales et al., 2006); (Rutkowski and Hegde, 2010); (Lin et al., 2008)

The ER transmembrane proteins; PERK, IRE1 $\alpha$ and ATF6 are the three main mammalian stress sensors and UPR initiators (Harding et al., 1999; Haze et al., 1999; Tirasophon et al., 1998). During ER stress, accumulation of misfolded proteins above a certain threshold causes the activation of stress sensors in an attempt to restore homeostasis. This is mainly achieved by decreasing the protein load that enters the ER, increasing ER biogenesis to efficiently handle misfoled proteins, facilitating the transport of damaged proteins to the cytosol for degradation and increasing the expression of UPR target genes encoding for protein folding chaperones (Ron and Walter, 2007; Shore et al., 2011). This corresponds to the adaptive output of the UPR and if this response is not sufficient to restore ER homeostasis, then the apoptotic response is triggered contributing to neuronal degeneration in different NDs (Ron and Walter, 2007; Shore et al., 2011). Chaperones carry out a multitude of homeostatic processes in the ER such as preventing the aggregation of proteins, refolding aberrantly folded proteins and disaggregating proteins aggregates (Hipp et al., 2014).Considering the critical role of chaperones in proteostasis, it is not surprising that in several NDs not only is there an accumulation of disease causing misfolded proteins, but these misfolded proteins also sequester chaperones such as Hsp70 and Hsp40, thereby depriving the ER of its main weapon against protein misfolding and proteostasis (Hetz and Mollereau, 2014).

\section{Autophagy-lysosome system}

Besides UPR, eukaryotic cells actively employ two other evolutionarily well conserved pathways for protein degradation, the ubiquitin-proteasome system and autophagylysosome pathway. Autophagy is a majorly lysosome-dependent protein degradation process, wherein cytosolic materials such as long lived proteins, aggregate-prone pathogenic proteins as well as damaged organelles are eliminated by enclosing them within double membrane vesicle termed as an "autophagosome". The autophagosome 
ultimately fuses with lysosome to degrade the isolated materials (Mizushima and Komatsu, 2011). Defective or insufficient regulation of the autophagy machinery and / or impairment of the lysosomal process can disrupt cellular homeostasis and lead to NDs including motoneuron degeneration (Shintani and Klionsky, 2004). Autophagy is classified into three major types: macroautophagy, microautophagy, and chaperonemediated autophagy (CMA). Macroautophagy (here onwards termed autophagy) is associated with the bulk degradation of cytoplasmic components via the formation of autopahgosomes, whereas microautophagy is a process that involves the direct uptake and degradation of cytoplasmic components by lysosomes, without the formation or involvement of transport vesicles (Glick et al., 2010; Mizushima and Komatsu, 2011). In the case of CMA, the presence of a consensus pentapeptide sequence, LysPheGluArgGln, is required in the substrate protein to which the chaperones Hsp70 binds, followed by the recognition of the substrate-chaperone complex by LAMP2A. The entire complex is then unfolded, moved across lysosomal membranes and eventually degraded within the lysosome (Arias and Cuervo, 2011; Cuervo, 2011; Kaushik et al., 2011). Considering the important role played by autophagy in the clearance of damaged or misfolded proteins, it is not surprising that deregulation of the autophagy pathway; involving insufficient activation of autophagy, impaired autophagic responses due to lysosomal dysfunction, and chronic autophagy activation induced cellular stress have been commonly observed in NDs (Cherra and Chu, 2008; Koga and Cuervo, 2011).

\section{Ubiquitin Proteasome System (UPS)}

UPS is a proteolytic mechanism whereby the conjugation of Ubiquitin (Ub) to specific substrates, lead to the degradation of the substrate via the proteasome (Ciechanover and Brundin, 2003; Ciechanover and Kwon, 2015). The process of substrate ubiquitination is an ATP-dependent enzymatic process involving Ub-activating enzyme $\mathrm{E} 1$, Ub-conjugating enzyme $\mathrm{E} 2$ and the $\mathrm{Ub}$ ligase $\mathrm{E} 3$, that specifically recognizes and mediates ubiquitination of substrates (Clague and Urbe, 2010). Upon ubiquitination of substrates at lysine 48 , the substrates are translocated to the $26 \mathrm{~S}$ proteasome. The $26 \mathrm{~S}$ proteasome is made up of a 20S core particle which is covered at both ends by a 19S regulatory subunit (Clague and Urbe, 2010). Normal substrates of the UPS pathway 
mainly include misfolded proteins, and short-lived proteins from all cellular compartments. The presence of abnormally folded proteins are recognized by chaperones and Ub ligases, which eventually trigger the UPS-associated degradation of those misfolded proteins (Ravid and Hochstrasser, 2008). In all major NDs, the pathology is associated with some form of dysfunction of the UPS pathway (Dennissen et al., 2012). As previously discussed, aging is a major risk factor, which actively contributes to the suboptimal functioning of the UPS system (Kikis et al., 2010). Agingassociated reduced degradation of misfolded proteins and the concomitant formation of pathological protein aggregates (Tydlacka et al., 2008). Furthermore, these insoluble aggegates inhibit the functioning of the UPS by sequestering important UPS components. Notably, pathogenic aggregates in NDs can directly inhibit proteasome activity (Cheroni et al., 2009; Kristiansen et al., 2007; Lindersson et al., 2004).

In this review, we discuss the different aspects of proteostasis impairment in NDs with a special focus on ALS. We explore the relationship between proteostasis impairment, protein aggregation and selective neuronal degeneration. We further examine the causal connection between the two processes and propose that targeting proteostasis impairment might provide a future therapeutic window for the treatment of NDs.

\section{Amyotrophic Lateral Sclerosis}

ALS, also known as Lou Gehrig's disease is the most common motoneuron disease with adult onset. It is a progressive, fatal neurodegenerative disease leading to the death of the patient within 3-5 years after diagnosis (Tandan and Bradley, 1985; Valdmanis and Rouleau, 2008). $10 \%$ of the ALS cases are genetic while the remaining $90 \%$ are sporadic but both forms of the disease are molecularly and clinically indistinguishable (Bosco and Landers, 2010; Gros-Louis et al., 2006). In inherited forms of ALS several genes that are mutated lead to the formation of abnormally folded proteins which aggregate and form inclusion bodies as well as actively impair proteostasis mechanisms (Soo et al., 2015). These are mainly SOD1, TAR DNA binding protein 43 (TDP-43), FUS, and C9ORF72 (Andersen and Al-Chalabi, 2011; DeJesus-Hernandez et al., 2011; Guo et al., 2010). Furthermore, dominant mutations in genes encoding for components of the protein quality control machinery related to vesicle transport, autophagy, ER 
homeostasis and UPS such as tubulin 4A (TUB4A), Dynein, Dynactin, sequestosome-1 (p62), Optineurin, valosin-containing protein (VCP or p97), vesicle-associated membrane protein associated protein B (VAPB), TANK1 binding kinase 1 (TBK1) and Ubiquilin-2 are all associated with ALS (Deng et al., 2011; Fecto et al., 2011; Freischmidt et al., 2015; Hipp et al., 2014; Johnson et al., 2010; Rademakers and van Blitterswijk, 2014; Smith et al., 2014; Synofzik et al., 2012; Williams et al., 2012).

Recently, hexanucleotide repeat expansion in the C9ORF72 gene was identified in patients displaying combined ALS / frontotemporal dementia (FTD) phenotype, thereby validating a genetic and a clinical overlap between these two diseases (Renton et al., 2011\{DeJesus-Hernandez, 2011 \#194)\}. The concept that ALS and FTLD exemplify the two ends of the same spectrum of disease was consolidated by the discovery of mutations within TDP-43 as being causally linked with sporadic and inherited forms of ALS as well as FTD. Furthermore, TDP-43 and fused in sarcoma FUS are present as pathological inclusions in the vast majority of superoxide dismutase 1 (SOD1) negative ALS cases as well as in subtypes of FTD thereby strengthening the notion that ALS and FTD belong to the same clinical spectrum of disease. Mutations in C9ORF72 gene are the most common mutation to be identified which is associated with FTD and / or ALS. A considerable effort has been put in understanding the pathological mechanism associated with the expanded C9ORF72 gene. Initial studies revealed a reduction in the mRNA levels of some C9ORF72 variants in ALS, suggesting a loss-of-function mechanism (DeJesus-Hernandez et al., 2011; Mori et al., 2013b; Renton et al., 2011). Pathomechanism involve the accumulation of transcripts containing hexanucleotide repeat expansion within the nucleus and forming toxic RNA foci (DeJesus-Hernandez et al., 2011). Presently, several studies have reported that the repeat-associated non-ATG (RAN) translation of the hexanucleotide expansion produces dipeptide-repeat proteins which forms neuronal inclusions and serves as a pathological hallmark of the disease (Ash et al., 2013; Mori et al., 2013a; Mori et al., 2013b).

Notably, all these mutations have been identified to directly target proteostasis network components and contribute to familial ALS, thereby providing direct evidence for the importance of proteostasis in ALS. However, it remains enigmatic how mutations in 
those proteins contribute to the disease. Majority of ALS patients present intracellular inclusions in degenerating neurons and glia cells (Wood et al., 2003), the composition of which is largely unknown, but several proteins have been identified such as Ubiquitin, Peripherin, Dorphin, SOD1 and TDP-43 (Leigh et al., 1991; Neumann et al., 2006; Niwa et al., 2002; Shibata et al., 1996). Additionally, these inclusions are also positive for components of the proteasome and neurofilaments (Hyun et al., 2003), implicating proteostasis impairment in ALS pathophysiology. Understanding whether those aggregates are protective in nature or whether they are the cause of neuronal death is crucial as this might impact future therapeutic strategies. Indeed, if those aggregates are self-protection mechanisms, their degradation once they are formed might actually lead to the worsening of the disease. In such cases, strategies where the stabilization of the nonpathogenic form of the protein is increased would be needed (Bosco et al., 2011).

\section{SOD1 and proteostasis impairment}

The first mutant protein associated with familial cases of ALS was superoxide dismutase 1 (SOD1) (Rosen et al., 1993) and to date over 170 different mutations have been found in ALS patients. Around $50 \%$ of the familial cases of ALS can be explained by mutations in either SOD1 or C9ORF72 (Leblond et al., 2014). ALS-causing SOD1 mutations do not influence the enzymatic activity of the protein (Valentine et al., 2005; Wong et al., 1995) but mutant SOD1 exhibits a high tendency to undergo conformational changes leading to aggregation of the protein (Bruijn et al., 1998; Chattopadhyay and Valentine, 2009; Furukawa et al., 2006; Molnar et al., 2009; Prudencio et al., 2009; Puttaparthi et al., 2004; Rakhit et al., 2004). The misfolded SOD1 protein seems to escape the normal degradation process through ubiquitination and has toxic effects on the degradation machinery, impairing the UPS pathway as well as autophagy (Bendotti et al., 2012; Chen et al., 2012; Cheroni et al., 2009; Robberecht and Philips, 2013). SOD1 knockout mice do not develop an ALS-like motor phenotype (Reaume et al., 1996), suggesting that conformation changes in SOD1 protein renders the enzyme cytotoxic via a gain of function mechanism (Andersen et al., 1995; Gaudette et al., 2000; Gurney et al., 1994). The importance of mutant SOD1 in the pathogenesis of ALS is confirmed by therapeutic approaches based on targeting the toxic protein species in mice, which have been 
successful in alleviating disease and expanding life span (Gros-Louis et al., 2010; Liu et al., 2012; Rakhit et al., 2007; Raoul et al., 2005; Urushitani et al., 2007).

The study of familial ALS using the well-established SOD1 mutant models enabled the identification and understanding of mechanisms and pathways that might be causally involved in the pathogenesis. Importantly, several studies identified that dysfunction of proteostasis leading to cellular stress plays a central role for the initiation and / or the progression of ALS (Bendotti et al., 2012; Saxena and Caroni, 2011). The burden of mutant SOD1 expression in motoneurons results in stress responses, which specifically leads to the activation of the unfolded protein response followed by microglial activation (Saxena et al., 2009). Interestingly, it was observed in sporadic patients that wild type SOD1 (WT-SOD1) undergoes age and environment associated modifications, causing conformational alterations in its structure. This leads to a gain of toxic properties similar to those observed in the case of mutant SOD1 (Bosco et al., 2010; Ezzi et al., 2007; Forsberg et al., 2010; Rakhit et al., 2002). Although the exact role WT-SOD1 plays in sporadic ALS cases still needs to be investigated, those data suggest that proteostasis impairment via SOD1 may be common a pathogenic mechanism between sporadic and familial ALS.

\section{Selective vulnerability of motoneuron subtype to UPR and axonal degeneration in SOD1 models of ALS}

Using the SOD1G93A mouse model, it was found that low-excitable fast fatiguable (FF) motoneurons are most vulnerable to denervation in ALS whereas medium excitable fatigue-resistant (FR) motoneurons and highly excitable slow (S) motoneurons resist denervation to a certain time period in the disease (Pun et al., 2006). This was an important finding in the field of ALS as excitability, which is a specific intrinsic characteristic of motoneuron subtypes could predict and determine the fate of the neuron in ALS. Notably, it was demonstrated that the vulnerable FF motoneurons were highly sensitive to chronic ER stress induced UPR, whereas the FR and S motoneurons were quasi resistant to ER stress (Saxena et al., 2009). Moreover, it was observed that ER stress is one of the earliest events detected in the asymptomatic phase of the 
disease and was restricted to vulnerable motoneurons and this response directly correlated with the disease associated stress burden (Saxena et al., 2009). Moreover, alterations in motoneuron excitability is an early process observed in mutant SOD1 expressing motoneurons as well as in both familial and sporadic ALS patients (Bories et al., 2007; Pambo-Pambo et al., 2009; Rothstein, 2009; Vucic et al., 2008), and it was recently shown that those excitability-related alterations governed impaired ER homeostasis in mutant SOD1 models (Saxena et al., 2013).

Recently, Filezac de L'Etang and colleagues identified SIL1, a BiP cochaperone which is required for protein translocation and folding in the ER (Anttonen et al., 2005), as being differentially expressed by the different sub-population of motoneurons. They found that the disease-resistant $S$ motoneurons present high levels of the cochaperone, whereas the ER stress-prone FF motoneurons expressed progressively and selectively reduced levels of SIL1. Using a mouse model of Marinesco-Sjögren syndrome, which exhibits a loss of function mutation in SIL1, it was demonstrated that SIL1 is crucial for the maintenance ER homeostasis in motoneurons. Indeed, when Sil1 heterozygous mice were crossed with SOD1G93A mice, authors found that SIL1 levels were progressively depleted in the FF motoneurons leading to enhanced ER stress and worsening of ALS pathology. Adeno-associated virus-mediated delivery of SIL1 in SOD1G93A mice restored ER homeostasis, delayed muscle denervation and prolonged the survival of the mice (Filezac de L'Etang et al., 2015)

An interesting study combining ALS patient derived induced Pluripotent stem cells (iPSCs) and RNA sequencing (RNA-seq), specifically examined the transcriptional and functional alterations brought about by the SOD1A4V mutation in human motoneurons. This study highlighted that mutant SOD1 selectively causes imbalance between ER stress and motoneuronal excitability (Kiskinis et al., 2014). Furthermore, iPSCs derived from patients having C9orf72 repeat expansions revealed that a conserved set of pathogenic changes such as ER stress casued by mutant SOD1 in human motoneurons are relevant to both familial and sporadic forms of ALS (Kiskinis et al., 2014). 


\section{Protein disulphide isomerases and their link to ALS}

It is intriguing how a cytosolic protein can induce stress within the ER. In fact, mutant SOD1 perturbs ER homeostasis at different levels leading to ER stress (Hetz and Mollereau, 2014). The transportation of mutant SOD1 from the ER to the Golgi is altered and it is known that mutant SOD1 oligomers localize to the ER lumen where they are linked to the chaperones protein disulphide isomerase and $\mathrm{BiP}$, resulting in the sequestration of those chaperones (Hetz and Mollereau, 2014; Kikuchi et al., 2006; Turner et al., 2005; Urushitani et al., 2006; Urushitani et al., 2008). Supporting this finding, several ER chaperones and Protein disulphide isomerase (PDI) were shown to be upregulated in ALS (Andreu et al., 2012; Massignan et al., 2007). For example, it was shown that PDI family members and ERp57 are upregulated in the spinal cord tissue of mutant SOD1 mice (Atkin et al., 2006; Atkin et al., 2008). Those findings were further confirmed in cerebrospinal fluid from sporadic ALS patients (Atkin et al., 2008). PDI family plays an important role in the protection against aggregation of mutant protein and inactivation of its enzymatic activity by S-nitrosylation results in loss of this protective activity in disease conditions (Walker, 2010; Walker et al., 2010). Hence, it is not surprising that mutation in PDI family members have been implicated in NDs where protein misfolding and aggregation is a central pathogenic mechanism (Perri et al., 2015). In 2015, Gonzalez-Perez and colleagues used a candidate gene approach and exome sequencing to search for mutations in the PDIA1 and PDIA3 or ERp57 that could be linked to ALS, and implicated 16 novel ALS-associated missense variants which might affect the catalytic function of PDIA1 or the Calreticulin / Calnexin cycle (Gonzalez-Perez et al., 2015). Recently, it was demonstrated that the enhanced expression of both wild-type PDIA1 and ERp57 in vitro enhances and accelerates dendritic outgrowth, a function which is lost in ALS-linked PDI mutants. Remarkably, Woehlbier et.al., in their recent study have shown that the defieciency of ERp57 specifically in the nervous system leads to deficits in motor performance, which is associated with the reduced expression of synaptic vesicle transporter protein and concomittant NMJ deficits. These finding demonstrated that loss of ERp57 function; a critical proteostasis component may underlie the pathogenic mechanism of those 
mutations and implicated ER proteostasis alterations in the etiology of ALS (Woehlbier et al., 2016)

\section{ER resident calcium-buffers: Calreticulin and Calnexin in SOD1 models of ALS}

The ER is the main intracellular calcium reservoir. The direct binding of calcium is needed for the correct function and optimal activity of several ER chaperones and foldases (Hetz and Mollereau, 2014), such as Calreticulin and Calnexin. Both these proteins function as molecular chaperones to assist in the folding and subunit assembly of the majority of Asn-linked glycoproteins that pass through the ER (Williams, 2006). Moreover, both Calnexin and Calreticulin have the ability to interact for extended durations with misfolded or incompletely assembled proteins, suggesting that they are components of the ER quality control system (David et al., 1993; Peterson et al., 1995) In 2012, it was shown that the vulnerable motoneurons in ALS present reduction in Calreticulin levels, triggering Fas activation which was sufficient to induce ER stress response in the vulnerable motoneurons and to trigger motoneuron death through the Fas / NO pathway (Bernard-Marissal et al., 2012). More recently, it was shown that deletion of a single Calreticulin allele in SOD1G93A mouse model exacerbated muscle weakness and denervation but did not influence motoneuron death (Bernard-Marissal et al., 2015), suggesting that impaired Calreticulin expression and function might be involved with the axonal pathology of ALS. Similarly, it was shown that Calnexin deficiency in mice lead to impairment in the structure of myelin sheets and to dysmyelination of the sciatic nerve and spinal cord (Kraus et al., 2010), implicating deficits or suboptimal fucntioning of Calreticulin and Calnexin in ALS pathomechanism.

\section{Modulating UPR pathways: targeting SOD1-induced motoneruon pathology}

Several studies that targeted the PERK branch of the UPR in the SOD1 mouse model highlighted a dual role of the pathway in disease. Indeed, on the one hand, it was found that treatment with Salubrinal, which is a specific inhibitor of elF2 $\alpha$ dephosphorylation (Boyce et al., 2005), protects motoneurons from ER stress (Saxena et al., 2009), and in vitro Salubrinal can decrease mutant SOD1 aggregation and cell death (Oh et al., 2008). On the other hand, deletion of its downstream transcription factor, ATF4 delays disease 
onset and prolongs life span in mutant SOD1 mice (Matus et al., 2013). Similarly, it was shown that deletion of X-box-binding protein-1 (XBP-1), an important UPR transcription factor involved in the regulation of protein folding and quality control genes enhanced the clearance of mutant SOD1 aggregates by macroautophagy (Hetz et al., 2009). Another mechanism by which mutant SOD1 can impact ER homeostasis, is via its aberrant association with Derlin-1, a component of the ER Associated Degradation (ERAD) machinery, which leads to its inhibition resulting in ER stress and proteostasis impairment (Nishitoh et al., 2008). Here, Derlin-1 overexpression was shown to be efficient in reducing the amount of accumulated mutant SOD1, leading to improved cell survival due to the inhibition of ER stress and concomitant activation of proteasomal and autophagy-associated degradation of mutant SOD1 (Mori et al., 2011).These findings further strengthen the notion that these pathways are complex modulators of cellular proteostasis involving active cross talks with different homeostasis-associated processes.

\section{Protein quality control (UPS and Autophagy) in SOD1-associated ALS:}

ER stress is not the only mechanisms that is involved in ALS associated proteostasis impairment, which is demonstrated by the fact that Salubrinal cannot completely cure SOD1G93A mice (Saxena et al., 2009). Other cellular processes such as altered RNA processing, pathological protein aggregation, mitochondrial dysfunction, axonal transport defects and glial cell dysfunction have been implicated in ALS pathogenesis (Andersen and Al-Chalabi, 2011; Boillée et al., 2006; Droppelmann et al., 2014; Ferraiuolo et al., 2011; Hetz and Mollereau, 2014; Lemmens et al., 2010; Robberecht and Philips, 2013; Saxena and Caroni, 2011; Schmidt et al., 2009; Strong, 2010). Impairment of the UPS (Cheroni et al., 2005; Cheroni et al., 2009) and the initiation of autophagy have been observed in SOD1G93A motoneurons (Hoffman et al., 1996; Li et al., 2008) as well as in spinal cord samples from ALS patients (Morimoto et al., 2007; Sasaki, 2011). It is not surprising that misregulation of autophagy and the excessive formation of autophagosomes has been observed in the spinal cord of sporadic ALS patients.(Morimoto et al., 2007). The appearance of autophagic dysfunction due to mutant SOD1-associated inclusions, actively impair protein quality control mechanisms 
by sequestering key components of the quality control machinery such as molecular chaperones Hsp70 and Hsp40, proteasomal subunits, Ub ligases: Dorfin and dynein required for cargo delivery to the aggresome (Di Noto et al., 2005; Hoffman et al., 1996). Both monomeric or oligomeric mutant SOD1 proteins also directly inhibit proteasomal activity (Hoffman et al., 1996; Hyun et al., 2003) and reduce autophagy-lysosome pathway (Zhang et al., 2011);.(Tokuda et al., 2016). Therefore, autophagy activators like trehalose (Gomes et al., 2010) and resveratrol (Kim et al., 2007) have been shown to have a beneficial effect on mutant SOD1 models of ALS. Moreover, reduced proteasomal activity also in turn promotes the accumulation of mutant SOD1 aggregates (Crippa et al., 2010). In the same line biochemical pathways such as retinoid pathways have also been linked to ALS pathology (Corcoran et al., 2002; Kolarcik and Bowser, 2012). It was for example shown that if one fed wild type rats with a retinoid-free diet, animals develop a phenotype that resembles ALS (Corcoran et al., 2002). Recently, it has been shown that retinoic acid can act on the UPS as well as on the autophagylysosome pathways helping to maintain proteostasis (Anguiano et al., 2013; Cheng et al., 2013; Rajawat et al., 2010; Riancho et al., 2015). Recently, Riancho et al., tested the effect of Bexarotene, a retinoid-X receptor agonist, in SODG93A mouse model and found that the drug significantly delayed motoneuron loss, motor symptoms and weight loss of the animals and extended life span of the mice (Riancho et al., 2015), thereby opening up new avenues for targeting proteostasis impairments. Thus, one mechanism underlying the pathogenesis of mutated SOD1-related ALS is a vicious cycle between misfolded proteins and proteolytic pathways, which accelerates the excessive accumulation of insoluble inclusions, leading to the death of affected motoneurons.

\section{Proteostasis impairment: TDP-43, FUS and other ALS-associated proteins}

TDP-43 is key pathological protein in ALS and FTD. Normal TDP-43 is a nuclear localized protein, that when mutated accumulates in the cytoplasm of ALS-affected motoneurons and ER stress has been shown to directly enhance the accumulation of mutant TDP-43 in the cytoplasm. ER stress was also shown to be responsible for the association of TDP-43 with stress granules, suggesting that ER stress is an important determinant of TDP-43 mediated pathogenicity (Walker et al., 2013). Moreover 
compounds that reduce ER stress such as salubrinal, guanabenz and phenazine serve as a strong suppressors of mutant TDP-43 toxicity. As these compounds target different branches of the ER stress signaling pathway, this implicates a prominent role for ER stress associated proteostasis impairment in TDP-43 related ALS (Vaccaro et al., 2013). Recently, it has been shown that inhibiting RAD-23; a dual component of the proteostasis machinery and nucleotide excision repair pathway, serves as a toxicity modifier for misfolded SOD1 and TDP-43. Interestingly, it was identified that increased expression of RAD-23 stabilized the misfolded TDP-43 proteins and altered their solubility and turnover rate by modifying the ubiquitination status of the mutant proteins (Jablonski et al., 2015). A direct for role TDP-43 in regulating autophagosomal and lysosomal biogenesis has recently been identified (Xia et al., 2016). Notably, both UPS and autophagy are actively involved in the clearance of TDP-43, which is dependent on the solubility of TDP-43. Soluble form of TDP-43 is preferentially degraded by the UPS, whereas the removal of oligomeric TDP-43 and TDP-43 aggregates is achieved via autophagy. Strikingly, it was found that functional UPS and autophagy was capable of clearing macroaggregates, which mimic the pathological features of the aggregates in patients (Scotter et al., 2014). Importantly, it was identified that the loss of TDP-43 led to the enhanced nuclear translocation of TFEB, thereby impairing the fusion of autophagosomes with lysosomes (Xia et al., 2016).

Similarly, normal FUS is located in the nucleus but in ALS, mutant FUS is sequestered outside the nucleus into the cytoplasm and forms cytoplasmic inclusions. The aberrant localization of mutant FUS to the cytoplasm leads to ER stress response and sequestration of an important chaperone PDI into inclusions. Notably, PDI and FUS positive inclusions have been observed in sporadic ALS and mutant FUS-related familial ALS patient tissues (Farg et al., 2012). Other ALS-associated proteins such as VCP function as an ATP-driven chaperon involved in the maturation of ubiquitin containing autophagosomes. Additionally, VCP plays an important role in the mitochondrial quality control, regulation of ER stress and autophagy. ALS causing VCP mutations lead to suboptimal functioning of these important quality control mechanisms thereby damaging proteostasis (Wojcik et al., 2006; Yamanaka et al., 2012). ALS8 caused by mutation in 
the VAPB gene have been implicated in ER stress and the impairment of proteasome function, thereby actively impairing protein homeostasis (Lev et al., 2008; Moumen et al., 2011). Similarly, loss of Optineurin function or ALS-causing Optineurin mutations increases protein aggregation, which in turn leads to the appearance of severe motor axonopathy. Furthermore, another ALS-associated protein TBK1 overlaped with Optineurin on protein aggregates and was primarily involved in aggregate removal via the autophagy-lysosome pathway (Korac et al., 2013). Moreover, mutations in Optineurin impairs autophagy-facilitated degradation of misfolded proteins as well as inhibits autophagosome formations, thereby contributing to proteostasis impairment and concomitant aggregate formation and accumulation (Shen et al., 2015). C9ORF72 has been shown to be involved in regulating endosomal trafficking and autophagy. Loss of C9ORF72 diminished the transport of proteins from the plasma membrane to the Golgi, and changed the ratio of autophagosome marker light chain 3 (LC3-II):(LC3-1), Notably, C9ORF72-related patient tissue presented enhanced overlap between C9ORF72, Rab7, and Rab11 proteins in motoneurons, thereby hinting towards trafficking and proteostasis deficits in C9ORF72 mediated ALS mechanism (Farg et al., 2014).

\section{Conclusions}

Impaired proteostasis has emerged as a key contributor to the pathophysiology of ALS. The current evidence for linking selective vulnerability of neurons in NDs suggests that important alterations in cellular homeostasis due to high accumulation of misfolded proteins can impair critical pathways required for the maintenance of proteostasis in those vulnerable neurons. Although studies in mouse models, in ALS patient tissues and iPSC derived motoneurons from patients indicate a link between proteostasis impairment and motoneuron degeneration, important causality issues remain to be addressed. These include the roles of increasing ER stress in influencing disease initiation, the synergistic influence of ER stress and misfolding protein accumulation in driving disease pathology, the role of irreversible UPR in engaging death signals, the role of autophagy and its recruitment and precise role in spatio-temporal aspects of disease and the cause for selective proteosomal deficts. Furthermore, the biological relevance and the specific contribution of the three branches of proteostasis and their 
regulation during normal and disease scenario needs to be further investigated and dissected. Over the years it has become clear that maintenance of proteostasis is a dynamic process involving network of signaling pathways, cross talk with other cellular processes and stress responses thereby enabling proper cell functioning.

\section{Figure Legend}

Fig. 1

Schematic depiction of normal cellular proteostasis and its impairment in neurodegenerative diseases (NDs)

In normal cells, the natural load of refolding and / or degradation of misfolded proteins is in balance with the cellular chaperone and degradative capacity. In NDs and aging, there is an increased load of misfolded mutant protein, leading to an imbalance in proteostasis and the accumulation of toxic protein aggregates. These aggregates in turn can sequester or saturate components of the protein quality control machinery. 


\section{References}

Alavez, S., et al., 2011. Amyloid-binding compounds maintain protein homeostasis during ageing and extend lifespan. Nature. 472, 226-9.

Alavez, S., Lithgow, G.J., 2012. Pharmacological maintenance of protein homeostasis could postpone age-related disease. Aging Cell. 11, 187-91.

Andersen, P.M., et al., 1995. Amyotrophic lateral sclerosis associated with homozygosity for an Asp90Ala mutation in CuZn-superoxide dismutase. Nature genetics. 10, 61-6.

Andersen, P.M., Al-Chalabi, A., 2011. Clinical genetics of amyotrophic lateral sclerosis: what do we really know? Nat Rev Neurol. 7, 603-15.

Andreu, C.I., et al., 2012. Protein disulfide isomerases in neurodegeneration: from disease mechanisms to biomedical applications. FEBS Lett. 586, 2826-34.

Anguiano, J., et al., 2013. Chemical modulation of chaperone-mediated autophagy by retinoic acid derivatives. Nat Chem Biol. 9, 374-82.

Anttonen, A.K., et al., 2005. The gene disrupted in Marinesco-Sjogren syndrome encodes SIL1, an HSPA5 cochaperone. Nat Genet. 37, 1309-11.

Arias, E., Cuervo, A.M., 2011. Chaperone-mediated autophagy in protein quality control. Curr Opin Cell Biol. 23, 184-9.

Ash, P.E., et al., 2013. Unconventional translation of C9ORF72 GGGGCC expansion generates insoluble polypeptides specific to c9FTD/ALS. Neuron. 77, 639-46.

Atkin, J.D., et al., 2006. Induction of the unfolded protein response in familial amyotrophic lateral sclerosis and association of protein-disulfide isomerase with superoxide dismutase 1 . J Biol Chem. 281, 30152-65.

Atkin, J.D., et al., 2008. Endoplasmic reticulum stress and induction of the unfolded protein response in human sporadic amyotrophic lateral sclerosis. Neurobiology of Disease. 30, 400-407.

Bendotti, C., et al., 2012. Dysfunction of constitutive and inducible ubiquitin-proteasome system in amyotrophic lateral sclerosis: implication for protein aggregation and immune response. Prog Neurobiol. 97, 101-26.

Bernales, S., Papa, F.R., Walter, P., 2006. Intracellular signaling by the unfolded protein response. Annu Rev Cell Dev Biol. 22, 487-508.

Bernard-Marissal, N., et al., 2012. Reduced calreticulin levels link endoplasmic reticulum stress and Fastriggered cell death in motoneurons vulnerable to ALS. J Neurosci. 32, 4901-12.

Bernard-Marissal, N., et al., 2015. Calreticulin levels determine onset of early muscle denervation by fast motoneurons of ALS model mice. Neurobiology of Disease. 73, 130-136.

Boillée, S., Vande Velde, C., Cleveland, Don W., 2006. ALS: A Disease of Motor Neurons and Their Nonneuronal Neighbors. Neuron. 52, 39-59. 
Bories, C., et al., 2007. Early electrophysiological abnormalities in lumbar motoneurons in a transgenic mouse model of amyotrophic lateral sclerosis. The European journal of neuroscience. 25, 451-9.

Bosco, D.A., Landers, J.E., 2010. Genetic determinants of amyotrophic lateral sclerosis as therapeutic targets. CNS Neurol Disord Drug Targets. 9, 779-90.

Bosco, D.A., et al., 2010. Wild-type and mutant SOD1 share an aberrant conformation and a common pathogenic pathway in ALS. Nat Neurosci. 13, 1396-403.

Bosco, D.A., et al., 2011. Proteostasis and movement disorders: Parkinson's disease and amyotrophic lateral sclerosis. Cold Spring Harb Perspect Biol. 3, a007500.

Boyce, M., et al., 2005. A selective inhibitor of elF2alpha dephosphorylation protects cells from ER stress. Science. 307, 935-9.

Brignull, H.R., et al., 2006. Polyglutamine proteins at the pathogenic threshold display neuron-specific aggregation in a pan-neuronal Caenorhabditis elegans model. The Journal of neuroscience : the official journal of the Society for Neuroscience. 26, 7597-606.

Bruijn, L.I., et al., 1998. Aggregation and motor neuron toxicity of an ALS-linked SOD1 mutant independent from wild-type SOD1. Science. 281, 1851-4.

Chattopadhyay, M., Valentine, J.S., 2009. Aggregation of copper-zinc superoxide dismutase in familial and sporadic ALS. Antioxid Redox Signal. 11, 1603-14.

Chen, S., et al., 2012. Autophagy dysregulation in amyotrophic lateral sclerosis. Brain Pathol. 22, 110-6.

Cheng, B., et al., 2013. Retinoic acid protects against proteasome inhibition associated cell death in SHSY5Y cells via the AKT pathway. Neurochem Int. 62, 31-42.

Cheroni, C., et al., 2005. Accumulation of human SOD1 and ubiquitinated deposits in the spinal cord of SOD1G93A mice during motor neuron disease progression correlates with a decrease of proteasome. Neurobiol Dis. 18, 509-22.

Cheroni, C., et al., 2009. Functional alterations of the ubiquitin-proteasome system in motor neurons of a mouse model of familial amyotrophic lateral sclerosis. Hum Mol Genet. 18, 82-96.

Cherra, S.J., 3rd, Chu, C.T., 2008. Autophagy in neuroprotection and neurodegeneration: A question of balance. Future Neurol. 3, 309-323.

Ciechanover, A., Brundin, P., 2003. The Ubiquitin Proteasome System in Neurodegenerative Diseases: Sometimes the Chicken, Sometimes the Egg. Neuron. 40, 427-446.

Ciechanover, A., Kwon, Y.T., 2015. Degradation of misfolded proteins in neurodegenerative diseases: therapeutic targets and strategies. Exp Mol Med. 47, e147.

Clague, M.J., Urbe, S., 2010. Ubiquitin: same molecule, different degradation pathways. Cell. 143, 682-5.

Corcoran, J., So, P.L., Maden, M., 2002. Absence of retinoids can induce motoneuron disease in the adult rat and a retinoid defect is present in motoneuron disease patients. J Cell Sci. 115, 4735-41. 
Crippa, V., et al., 2010. The small heat shock protein B8 (HspB8) promotes autophagic removal of misfolded proteins involved in amyotrophic lateral sclerosis (ALS). Hum Mol Genet. 19, 3440-56.

Cuanalo-Contreras, K., Mukherjee, A., Soto, C., 2013. Role of protein misfolding and proteostasis deficiency in protein misfolding diseases and aging. Int J Cell Biol. 2013, 638083.

Cuervo, A.M., 2011. Chaperone-mediated autophagy: Dice's 'wild' idea about lysosomal selectivity. Nat Rev Mol Cell Biol. 12, 535-41.

David, D.C., et al., 2010. Widespread protein aggregation as an inherent part of aging in C. elegans. PLoS Biol. 8, e1000450.

David, V., et al., 1993. Interaction with newly synthesized and retained proteins in the endoplasmic reticulum suggests a chaperone function for human integral membrane protein IP90 (calnexin). J Biol Chem. 268, 9585-92.

Davies, S.W., et al., 1997. Formation of neuronal intranuclear inclusions underlies the neurological dysfunction in mice transgenic for the HD mutation. Cell. 90, 537-48.

DeJesus-Hernandez, M., et al., 2011. Expanded GGGGCC hexanucleotide repeat in noncoding region of C9ORF72 causes chromosome 9p-linked FTD and ALS. Neuron. 72, 245-56.

Deng, H.X., et al., 2011. Mutations in UBQLN2 cause dominant X-linked juvenile and adult-onset ALS and ALS/dementia. Nature. 477, 211-5.

Dennissen, F.J., Kholod, N., van Leeuwen, F.W., 2012. The ubiquitin proteasome system in neurodegenerative diseases: culprit, accomplice or victim? Prog Neurobiol. 96, 190-207.

Di Noto, L., et al., 2005. Proteasomal degradation of mutant superoxide dismutases linked to amyotrophic lateral sclerosis. J Biol Chem. 280, 39907-13.

Droppelmann, C.A., et al., 2014. The emerging role of guanine nucleotide exchange factors in ALS and other neurodegenerative diseases. Frontiers in cellular neuroscience. 8, 282.

Ezzi, S.A., Urushitani, M., Julien, J.P., 2007. Wild-type superoxide dismutase acquires binding and toxic properties of ALS-linked mutant forms through oxidation. J Neurochem. 102, 170-8.

Farg, M.A., et al., 2012. Mutant FUS induces endoplasmic reticulum stress in amyotrophic lateral sclerosis and interacts with protein disulfide-isomerase. Neurobiol Aging. 33, 2855-68.

Farg, M.A., et al., 2014. C9ORF72, implicated in amytrophic lateral sclerosis and frontotemporal dementia, regulates endosomal trafficking. Hum Mol Genet. 23, 3579-95.

Fecto, F., et al., 2011. SQSTM1 mutations in familial and sporadic amyotrophic lateral sclerosis. Arch Neurol. 68, 1440-6.

Ferraiuolo, L., et al., 2011. Molecular pathways of motor neuron injury in amyotrophic lateral sclerosis. Nature reviews. Neurology. 7, 616-30.

Filezac de L'Etang, A., et al., 2015. Marinesco-Sjogren syndrome protein SIL1 regulates motor neuron subtype-selective ER stress in ALS. Nat Neurosci. 18, 227-38. 
Forsberg, K., et al., 2010. Novel antibodies reveal inclusions containing non-native SOD1 in sporadic ALS patients. PLoS One. 5, e11552.

Freischmidt, A., et al., 2015. Haploinsufficiency of TBK1 causes familial ALS and fronto-temporal dementia. Nat Neurosci. 18, 631-6.

Frickel, E.M., et al., 2004. ERp57 is a multifunctional thiol-disulfide oxidoreductase. J Biol Chem. 279, 18277-87.

Frost, B., Diamond, M.I., 2010. Prion-like mechanisms in neurodegenerative diseases. Nature reviews. Neuroscience. 11, 155-9.

Furukawa, Y., et al., 2006. Disulfide cross-linked protein represents a significant fraction of ALSassociated $\mathrm{Cu}, \mathrm{Zn}$-superoxide dismutase aggregates in spinal cords of model mice. Proc Natl Acad Sci U S A. 103, 7148-53.

Gaudette, M., Hirano, M., Siddique, T., 2000. Current status of SOD1 mutations in familial amyotrophic lateral sclerosis. Amyotrophic lateral sclerosis and other motor neuron disorders : official publication of the World Federation of Neurology, Research Group on Motor Neuron Diseases. 1, 83-9.

Gidalevitz, T., et al., 2006. Progressive disruption of cellular protein folding in models of polyglutamine diseases. Science. 311, 1471-4.

Glick, D., Barth, S., Macleod, K.F., 2010. Autophagy: cellular and molecular mechanisms. J Pathol. 221, 312.

Golde, T.E., Miller, V.M., 2009. Proteinopathy-induced neuronal senescence: a hypothesis for brain failure in Alzheimer's and other neurodegenerative diseases. Alzheimer's research \& therapy. 1, 5.

Gomes, C., Escrevente, C., Costa, J., 2010. Mutant superoxide dismutase 1 overexpression in NSC-34 cells: effect of trehalose on aggregation, TDP-43 localization and levels of co-expressed glycoproteins. Neurosci Lett. 475, 145-9.

Gonzalez-Perez, P., et al., 2015. Identification of rare protein disulfide isomerase gene variants in amyotrophic lateral sclerosis patients. Gene. 566, 158-65.

Gros-Louis, F., Gaspar, C., Rouleau, G.a., 2006. Genetics of familial and sporadic amyotrophic lateral sclerosis. Biochimica et biophysica acta. 1762, 956-972.

Gros-Louis, F., et al., 2010. Intracerebroventricular infusion of monoclonal antibody or its derived Fab fragment against misfolded forms of SOD1 mutant delays mortality in a mouse model of ALS. Journal of neurochemistry. 113, 1188-99.

Guo, Y., et al., 2010. Ultrastructural diversity of inclusions and aggregations in the lumbar spinal cord of SOD1-G93A transgenic mice. Brain Res. 1353, 234-44.

Gurney, M.E., et al., 1994. Motor neuron degeneration in mice that express a human $\mathrm{Cu}, \mathrm{Zn}$ superoxide dismutase mutation. Science. 264, 1772-5. 
Haass, C., Selkoe, D.J., 2007. Soluble protein oligomers in neurodegeneration: lessons from the Alzheimer's amyloid beta-peptide. Nature reviews. Molecular cell biology. 8, 101-12.

Harding, H.P., Zhang, Y., Ron, D., 1999. Protein translation and folding are coupled by an endoplasmicreticulum-resident kinase. Nature. 397, 271-4.

Hartl, F.U., Bracher, A., Hayer-Hartl, M., 2011. Molecular chaperones in protein folding and proteostasis. Nature. 475, 324-32.

Haze, K., et al., 1999. Mammalian transcription factor ATF6 is synthesized as a transmembrane protein and activated by proteolysis in response to endoplasmic reticulum stress. Mol Biol Cell. 10, 378799.

Hetz, C., et al., 2009. XBP-1 deficiency in the nervous system protects against amyotrophic lateral sclerosis by increasing autophagy. Genes Dev. 23, 2294-306.

Hetz, C., Mollereau, B., 2014. Disturbance of endoplasmic reticulum proteostasis in neurodegenerative diseases. Nat Rev Neurosci. 15, 233-49.

Hetz, C., Chevet, E., Oakes, S.A., 2015. Proteostasis control by the unfolded protein response. Nat Cell Biol. 17, 829-38.

Hipp, M.S., Park, S.-H., Hartl, F.U., 2014. Proteostasis impairment in protein-misfolding and -aggregation diseases. Trends in Cell Biology. 24, 506-514.

Hoffman, E.K., et al., 1996. Proteasome inhibition enhances the stability of mouse Cu/Zn superoxide dismutase with mutations linked to familial amyotrophic lateral sclerosis. J Neurol Sci. 139, 1520.

Hyun, D.H., et al., 2003. Proteasomal inhibition causes the formation of protein aggregates containing a wide range of proteins, including nitrated proteins. J Neurochem. 86, 363-73.

Jablonski, A.M., et al., 2015. Loss of RAD-23 Protects Against Models of Motor Neuron Disease by Enhancing Mutant Protein Clearance. J Neurosci. 35, 14286-306.

Johnson, J.O., et al., 2010. Exome sequencing reveals VCP mutations as a cause of familial ALS. Neuron. $68,857-64$.

Kaushik, S., et al., 2011. Chaperone-mediated autophagy at a glance. J Cell Sci. 124, 495-9.

Kikis, E.A., Gidalevitz, T., Morimoto, R.I., 2010. Protein homeostasis in models of aging and age-related conformational disease. Adv Exp Med Biol. 694, 138-59.

Kikuchi, H., et al., 2006. Spinal cord endoplasmic reticulum stress associated with a microsomal accumulation of mutant superoxide dismutase-1 in an ALS model. Proc Natl Acad Sci U S A. 103, 6025-30.

Kim, Y.A., et al., 2007. Resveratrol inhibits nitric oxide and prostaglandin E2 production by lipopolysaccharide-activated C6 microglia. J Med Food. 10, 218-24. 
Kiskinis, E., et al., 2014. Pathways disrupted in human ALS motor neurons identified through genetic correction of mutant SOD1. Cell Stem Cell. 14, 781-95.

Koga, H., Cuervo, A.M., 2011. Chaperone-mediated autophagy dysfunction in the pathogenesis of neurodegeneration. Neurobiol Dis. 43, 29-37.

Kolarcik, C.L., Bowser, R., 2012. Retinoid signaling alterations in amyotrophic lateral sclerosis. Am J Neurodegener Dis. 1, 130-45.

Koo, E.H., Lansbury, P.T., Kelly, J.W., 1999. Amyloid diseases: abnormal protein aggregation in neurodegeneration. Proceedings of the National Academy of Sciences of the United States of America. 96, 9989-90.

Korac, J., et al., 2013. Ubiquitin-independent function of optineurin in autophagic clearance of protein aggregates. J Cell Sci. 126, 580-92.

Kraus, A., et al., 2010. Calnexin deficiency leads to dysmyelination. J Biol Chem. 285, 18928-38.

Kristiansen, M., et al., 2007. Disease-associated prion protein oligomers inhibit the 265 proteasome. Mol Cell. 26, 175-88.

Leblond, C.S., et al., 2014. Dissection of genetic factors associated with amyotrophic lateral sclerosis. Exp Neurol. 262 Pt B, 91-101.

Leigh, P.N., et al., 1991. Ubiquitin-immunoreactive intraneuronal inclusions in amyotrophic lateral sclerosis. Morphology, distribution, and specificity. Brain : a journal of neurology. 114 ( Pt 2, 775788.

Lemmens, R., et al., 2010. RNA metabolism and the pathogenesis of motor neuron diseases. Trends Neurosci. 33, 249-58.

Lev, S., et al., 2008. The VAP protein family: from cellular functions to motor neuron disease. Trends Cell Biol. 18, 282-90.

Li, L., Zhang, X., Le, W., 2008. Altered macroautophagy in the spinal cord of SOD1 mutant mice. Autophagy. 4, 290-3.

Lin, J.H., Walter, P., Yen, T.S., 2008. Endoplasmic reticulum stress in disease pathogenesis. Annu Rev Pathol. 3, 399-425.

Lindersson, E., et al., 2004. Proteasomal inhibition by alpha-synuclein filaments and oligomers. J Biol Chem. 279, 12924-34.

Liu, H.-N., et al., 2012. Targeting of monomer/misfolded SOD1 as a therapeutic strategy for amyotrophic lateral sclerosis. The Journal of neuroscience : the official journal of the Society for Neuroscience. $32,8791-9$.

Martindale, D., et al., 1998. Length of huntingtin and its polyglutamine tract influences localization and frequency of intracellular aggregates. Nature genetics. 18, 150-4. 
Massignan, T., et al., 2007. Proteomic analysis of spinal cord of presymptomatic amyotrophic lateral sclerosis G93A SOD1 mouse. Biochemical and Biophysical Research Communications. 353, 719725.

Matus, S., et al., 2013. Functional contribution of the transcription factor ATF4 to the pathogenesis of amyotrophic lateral sclerosis. PLoS One. 8, e66672.

Mizushima, N., Komatsu, M., 2011. Autophagy: renovation of cells and tissues. Cell. 147, 728-41.

Molnar, K.S., et al., 2009. A common property of amyotrophic lateral sclerosis-associated variants: destabilization of the copper/zinc superoxide dismutase electrostatic loop. J Biol Chem. 284, 30965-73.

Mori, A., et al., 2011. Derlin-1 overexpression ameliorates mutant SOD1-induced endoplasmic reticulum stress by reducing mutant SOD1 accumulation. Neurochem Int. 58, 344-53.

Mori, K., et al., 2013a. Bidirectional transcripts of the expanded C9orf72 hexanucleotide repeat are translated into aggregating dipeptide repeat proteins. Acta Neuropathol. 126, 881-93.

Mori, K., et al., 2013b. The C9orf72 GGGGCC repeat is translated into aggregating dipeptide-repeat proteins in FTLD/ALS. Science. 339, 1335-8.

Morimoto, N., et al., 2007. Increased autophagy in transgenic mice with a G93A mutant SOD1 gene. Brain Res. 1167, 112-7.

Moumen, A., Virard, I., Raoul, C., 2011. Accumulation of wildtype and ALS-linked mutated VAPB impairs activity of the proteasome. PLoS One. 6, e26066.

Neumann, M., et al., 2006. Ubiquitinated TDP-43 in frontotemporal lobar degeneration and amyotrophic lateral sclerosis. Science (New York, N.Y.). 314, 130-3.

Nishitoh, H., et al., 2008. ALS-linked mutant SOD1 induces ER stress- and ASK1-dependent motor neuron death by targeting Derlin-1. Genes Dev. 22, 1451-64.

Niwa, J.I., et al., 2002. Dorfin ubiquitylates mutant SOD1 and prevents mutant SOD1-mediated neurotoxicity. Journal of Biological Chemistry. 277, 36793-36798.

Oh, Y.K., et al., 2008. Superoxide dismutase 1 mutants related to amyotrophic lateral sclerosis induce endoplasmic stress in neuro2a cells. J Neurochem. 104, 993-1005.

Oliver, J.D., et al., 1997. Interaction of the thiol-dependent reductase ERp57 with nascent glycoproteins. Science. 275, 86-8.

Oliver, J.D., et al., 1999. ERp57 functions as a subunit of specific complexes formed with the ER lectins calreticulin and calnexin. Mol Biol Cell. 10, 2573-82.

Pambo-Pambo, A., Durand, J., Gueritaud, J.-P., 2009. Early excitability changes in lumbar motoneurons of transgenic SOD1G85R and SOD1G(93A-Low) mice. Journal of neurophysiology. 102, 3627-42.

Perri, E.R., et al., 2015. The Unfolded Protein Response and the Role of Protein Disulfide Isomerase in Neurodegeneration. Front Cell Dev Biol. 3, 80. 
Peterson, J.R., et al., 1995. Transient, lectin-like association of calreticulin with folding intermediates of cellular and viral glycoproteins. Mol Biol Cell. 6, 1173-84.

Prudencio, M., et al., 2009. Variation in aggregation propensities among ALS-associated variants of SOD1: correlation to human disease. Hum Mol Genet. 18, 3217-26.

Pun, S., et al., 2006. Selective vulnerability and pruning of phasic motoneuron axons in motoneuron disease alleviated by CNTF. Nature neuroscience. 9, 408-19.

Puttaparthi, K., et al., 2004. Aggregate formation in the spinal cord of mutant SOD1 transgenic mice is reversible and mediated by proteasomes. Journal of Neurochemistry. 87, 851-860.

Rademakers, R., van Blitterswijk, M., 2014. Excess of rare damaging TUBA4A variants suggests cytoskeletal defects in ALS. Neuron. 84, 241-3.

Rajawat, Y., Hilioti, Z., Bossis, I., 2010. Autophagy: a target for retinoic acids. Autophagy. 6, 1224-6.

Rakhit, R., et al., 2002. Oxidation-induced misfolding and aggregation of superoxide dismutase and its implications for amyotrophic lateral sclerosis. J Biol Chem. 277, 47551-6.

Rakhit, R., et al., 2004. Monomeric $\mathrm{Cu}, \mathrm{Zn}$-superoxide dismutase is a common misfolding intermediate in the oxidation models of sporadic and familial amyotrophic lateral sclerosis. The Journal of biological chemistry. 279, 15499-504.

Rakhit, R., et al., 2007. An immunological epitope selective for pathological monomer-misfolded SOD1 in ALS. Nature medicine. 13, 754-9.

Raoul, C., et al., 2005. Lentiviral-mediated silencing of SOD1 through RNA interference retards disease onset and progression in a mouse model of ALS. Nature medicine. 11, 423-8.

Ravid, T., Hochstrasser, M., 2008. Diversity of degradation signals in the ubiquitin-proteasome system. Nat Rev Mol Cell Biol. 9, 679-90.

Reaume, A.G., et al., 1996. Motor neurons in Cu/Zn superoxide dismutase-deficient mice develop normally but exhibit enhanced cell death after axonal injury. Nature genetics. 13, 43-7.

Reis-Rodrigues, P., et al., 2012. Proteomic analysis of age-dependent changes in protein solubility identifies genes that modulate lifespan. Aging Cell. 11, 120-7.

Renton, A.E., et al., 2011. A hexanucleotide repeat expansion in C9ORF72 is the cause of chromosome 9p21-linked ALS-FTD. Neuron. 72, 257-68.

Riancho, J., et al., 2015. Neuroprotective Effect of Bexarotene in the SOD1(G93A) Mouse Model of Amyotrophic Lateral Sclerosis. Front Cell Neurosci. 9, 250.

Robberecht, W., Philips, T., 2013. The changing scene of amyotrophic lateral sclerosis. Nature reviews. Neuroscience. 14, 248-64.

Ron, D., Walter, P., 2007. Signal integration in the endoplasmic reticulum unfolded protein response. Nat Rev Mol Cell Biol. 8, 519-529. 
Rosen, D.R., et al., 1993. Mutations in Cu/Zn superoxide dismutase gene are associated with familial amyotrophic lateral sclerosis. Nature. 362, 59-62.

Roth, D.M., Balch, W.E., 2011. Modeling general proteostasis: proteome balance in health and disease. Curr Opin Cell Biol. 23, 126-34.

Rothstein, J.D., 2009. Current hypotheses for the underlying biology of amyotrophic lateral sclerosis. Annals of neurology. 65 Suppl 1, S3-9.

Rutkowski, D.T., Hegde, R.S., 2010. Regulation of basal cellular physiology by the homeostatic unfolded protein response. J Cell Biol. 189, 783-94.

Sasaki, S., 2011. Autophagy in spinal cord motor neurons in sporadic amyotrophic lateral sclerosis. J Neuropathol Exp Neurol. 70, 349-59.

Saxena, S., Cabuy, E., Caroni, P., 2009. A role for motoneuron subtype-selective ER stress in disease manifestations of FALS mice. Nature neuroscience. 12, 627-36.

Saxena, S., Caroni, P., 2011. Selective neuronal vulnerability in neurodegenerative diseases: from stressor thresholds to degeneration. Neuron. 71, 35-48.

Saxena, S., et al., 2013. Neuroprotection through excitability and mTOR required in ALS motoneurons to delay disease and extend survival. Neuron. 80, 80-96.

Schmidt, E.R., Pasterkamp, R.J., van den Berg, L.H., 2009. Axon guidance proteins: novel therapeutic targets for ALS? Prog Neurobiol. 88, 286-301.

Schubert, U., et al., 2000. Rapid degradation of a large fraction of newly synthesized proteins by proteasomes. Nature. 404, 770-4.

Scotter, E.L., et al., 2014. Differential roles of the ubiquitin proteasome system and autophagy in the clearance of soluble and aggregated TDP-43 species. J Cell Sci. 127, 1263-78.

Shen, W.C., et al., 2015. Mutations in the ubiquitin-binding domain of OPTN/optineurin interfere with autophagy-mediated degradation of misfolded proteins by a dominant-negative mechanism. Autophagy. 11, 685-700.

Shibata, N., et al., 1996. Intense superoxide dismutase-1 immunoreactivity in intracytoplasmic hyaline inclusions of familial amyotrophic lateral sclerosis with posterior column involvement. Journal of neuropathology and experimental neurology. 55, 481-490.

Shintani, T., Klionsky, D.J., 2004. Autophagy in health and disease: a double-edged sword. Science. 306, 990-5.

Shore, G.C., Papa, F.R., Oakes, S.A., 2011. Signaling cell death from the endoplasmic reticulum stress response. Current Opinion in Cell Biology. 23, 143-149.

Smith, B.N., et al., 2014. Exome-wide rare variant analysis identifies TUBA4A mutations associated with familial ALS. Neuron. 84, 324-31. 
Soo, K.Y., et al., 2015. Rab1-dependent ER-Golgi transport dysfunction is a common pathogenic mechanism in SOD1, TDP-43 and FUS-associated ALS. Acta Neuropathol. 130, 679-97.

Stoppini, M., et al., 2004. Neurodegenerative diseases caused by protein aggregation: a phenomenon at the borderline between molecular evolution and ageing. Pharmacological research : the official journal of the Italian Pharmacological Society. 50, 419-31.

Strong, M.J., 2010. The evidence for altered RNA metabolism in amyotrophic lateral sclerosis (ALS). Journal of the neurological sciences. 288, 1-12.

Synofzik, M., et al., 2012. Screening in ALS and FTD patients reveals 3 novel UBQLN2 mutations outside the PXX domain and a pure FTD phenotype. Neurobiol Aging. 33, 2949 e13-7.

Takalo, M., et al., 2013. Protein aggregation and degradation mechanisms in neurodegenerative diseases. American journal of neurodegenerative disease. 2, 1-14.

Tandan, R., Bradley, W.G., 1985. Amyotrophic lateral sclerosis: Part 1. Clinical features, pathology, and ethical issues in management. Annals of neurology. 18, 271-280.

Tirasophon, W., Welihinda, A.A., Kaufman, R.J., 1998. A stress response pathway from the endoplasmic reticulum to the nucleus requires a novel bifunctional protein kinase/endoribonuclease (Ire1p) in mammalian cells. Genes Dev. 12, 1812-24.

Tokuda, E., et al., 2016. Low autophagy capacity implicated in motor system vulnerability to mutant superoxide dismutase. Acta Neuropathol Commun. 4, 6.

Turner, B.J., et al., 2005. Impaired extracellular secretion of mutant superoxide dismutase 1 associates with neurotoxicity in familial amyotrophic lateral sclerosis. J Neurosci. 25, 108-17.

Tydlacka, S., et al., 2008. Differential activities of the ubiquitin-proteasome system in neurons versus glia may account for the preferential accumulation of misfolded proteins in neurons. J Neurosci. 28, 13285-95.

Urushitani, M., et al., 2006. Chromogranin-mediated secretion of mutant superoxide dismutase proteins linked to amyotrophic lateral sclerosis. Nat Neurosci. 9, 108-18.

Urushitani, M., Ezzi, S.A., Julien, J.-P., 2007. Therapeutic effects of immunization with mutant superoxide dismutase in mice models of amyotrophic lateral sclerosis. Proceedings of the National Academy of Sciences of the United States of America. 104, 2495-500.

Urushitani, M., et al., 2008. The endoplasmic reticulum-Golgi pathway is a target for translocation and aggregation of mutant superoxide dismutase linked to ALS. Faseb j. 22, 2476-87.

Vaccaro, A., et al., 2013. Pharmacological reduction of ER stress protects against TDP-43 neuronal toxicity in vivo. Neurobiol Dis. 55, 64-75.

Valdmanis, P.N., Rouleau, G.a., 2008. Genetics of familial amyotrophic lateral sclerosis. Neurology. 70, 144-152.

Valentine, J.S., Doucette, P.A., Zittin Potter, S., 2005. Copper-zinc superoxide dismutase and amyotrophic lateral sclerosis. Annu Rev Biochem. 74, 563-93. 
Vucic, S., Nicholson, G.A., Kiernan, M.C., 2008. Cortical hyperexcitability may precede the onset of familial amyotrophic lateral sclerosis. Brain. 131, 1540-1550.

Walker, A.K., 2010. Protein disulfide isomerase and the endoplasmic reticulum in amyotrophic lateral sclerosis. J Neurosci. 30, 3865-7.

Walker, A.K., et al., 2010. Protein disulphide isomerase protects against protein aggregation and is Snitrosylated in amyotrophic lateral sclerosis. Brain. 133, 105-16.

Walker, A.K., et al., 2013. ALS-associated TDP-43 induces endoplasmic reticulum stress, which drives cytoplasmic TDP-43 accumulation and stress granule formation. PLoS One. 8, e81170.

Williams, D.B., 2006. Beyond lectins: the calnexin/calreticulin chaperone system of the endoplasmic reticulum. J Cell Sci. 119, 615-23.

Williams, K.L., et al., 2012. UBQLN2/ubiquilin 2 mutation and pathology in familial amyotrophic lateral sclerosis. Neurobiol Aging. 33, 2527 e3-10.

Woehlbier, U., et al., 2016. ALS-linked protein disulfide isomerase variants cause motor dysfunction. EMBO J.

Wojcik, C., et al., 2006. Valosin-containing protein (p97) is a regulator of endoplasmic reticulum stress and of the degradation of $\mathrm{N}$-end rule and ubiquitin-fusion degradation pathway substrates in mammalian cells. Mol Biol Cell. 17, 4606-18.

Wong, P.C., et al., 1995. An adverse property of a familial ALS-linked SOD1 mutation causes motor neuron disease characterized by vacuolar degeneration of mitochondria. Neuron. 14, 1105-16.

Wood, J.D., Beaujeux, T.P., Shaw, P.J., 2003. Protein aggregation in motor neurone disorders. In: Neuropathology and Applied Neurobiology. Vol. 29, ed.^eds., pp. 529-545.

Xia, Q., et al., 2016. TDP-43 loss of function increases TFEB activity and blocks autophagosome-lysosome fusion. EMBO J. 35, 121-42.

Yamanaka, K., Sasagawa, Y., Ogura, T., 2012. Recent advances in p97/VCP/Cdc48 cellular functions. Biochim Biophys Acta. 1823, 130-7.

Zhang, X., et al., 2011. Rapamycin treatment augments motor neuron degeneration in SOD1(G93A) mouse model of amyotrophic lateral sclerosis. Autophagy. 7, 412-25. 
NDs such as ALS / Aging

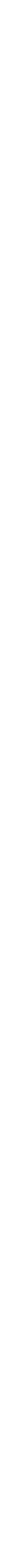

